\title{
Promising outcomes of a national programme for the prevention of mother-to-child HIV transmission in Addis Ababa: a retrospective study
}

\author{
Alemnesh H Mirkuzie ${ }^{1,2^{*}}$, Sven Gudmund Hinderaker ${ }^{1}$, Odd Mørkve
}

\begin{abstract}
Background: Prevention of Mother-to-Child HIV Transmission (PMTCT) is still the most effective intervention in combating new HIV infections. In 2008, revised national PMTCT guidelines that incorporated new policies on HIV counselling and testing, antiretroviral prophylaxis regimen and infant HIV diagnosis came into effect in Ethiopia. In the present study we have examined trends in PMTCT service utilization and assessed the rate of MTCT in relation to policy changes in the national PMTCT programme.
\end{abstract}

Methods: Reports from February 2004 to August 2009 were reviewed in 10 sub-cities in Addis Ababa, Ethiopia. The data was collected from May to October 2009.

Results: The proportion of women who received HIV counselling and testing among new antenatal care attendees increased from $50.7 \%$ (95\% Cl 50.2-51.2) in 2007 to $84.5 \%$ (95\% Cl 84.1-84.9) in 2009 following the shift to routine opt-out testing. Nevertheless, in 2009 only $53.7 \%$ of the positive women and $40.7 \%$ of their infants received antiretroviral prophylaxis. The HIV prevalence among antenatal attendees decreased significantly from $10.5 \%$ in 2004 to $4.6 \%$ in 2009 in parallel to the increased number of women being tested. The HIV positive women were over 18 times (RR 18.5, p < 0.0001) more likely to be referred for treatment, care and support in 2009 than in 2004. The proportion of partners tested for HIV decreased by 14\% in 2009 compared to 2004, although the absolute number was increasing year by year. Only $10.6 \%$ (95\% CI 9.9-11.2) of the HIV positive women completed their follow up to infant HIV testing. The cumulative probability of HIV infection among babies on single dose nevirapine regimen who were tested at $>=18$ months was $15.0 \%$ (95\% Cl 9.8-22.1) in 2007, whereas it was 8.2\% (95\% Cl 5.55-11.97) among babies on Zidovudine regimen who were tested at >=45 days in 2009.

Conclusion: The paper demonstrates trends in PMTCT service utilization in relation to changing policy. There is marked improvement in HIV counselling and testing service utilization, especially after the policy shift to routine opt-out testing. However, despite policy changes, the ARV prophylaxis uptake, the loss to follow up and the partner testing have remained unchanged across the years. This should be a matter of immediate concern and a topic for further research.

\section{Background}

Prevention of mother-to-child HIV transmission (PMTCT) is still the most effective intervention in combating new HIV infections [1]. When the possibility of having an efficacious vaccine seems questionable, as

\footnotetext{
* Correspondence: ami048@cih.uib.no

${ }^{1}$ Centre for International Health, University of Bergen, Overlege Danielssens Hus, Årstav. 21, Bergen 5020, Norway

Full list of author information is available at the end of the article
}

reported in a recent vaccine trial, holding on to PMTCT programmes gives some hope [2]. PMTCT is a multifaceted intervention. It is not just a way to stop vertical transmission of HIV but also to provide access to treatment, care and support for women who would otherwise not get the chance to know their HIV status before it is too late [1].

Despite its importance, a PMTCT programme often suffers poor resource allocation that could threaten 
programme success [3]. Globally, the challenges in PMTCT programme implementation combined with ever changing scientific advances call for frequent revisits of policies and strategies. In Ethiopia, the first PMTCT guidelines were developed in 2001, incorporating early recommendations by the WHO on HIV counselling and testing, ARV prophylaxis regimen, infant feeding counselling, infant HIV diagnosis algorithm, partner testing and referring HIV positive pregnant women for treatment, care and support [4]. During the recent years, the WHO has made several policy changes to improve PMTCT programme performance. In 2004, the HIV counselling and testing policy was shifted from client initiated opt-in approach to routine opt-out approach in order to improve women's access to prevention interventions and to contain HIV testing within the standard of care for pregnant women [5]. In 2006, the antiretroviral (ARV) prophylaxis regimen was changed from short course single dose NVP (sdNVP) to a more efficacious multidrug zidovudine (ZDV) regimen $[1,6]$. The infant feeding recommendation was also revised in 2006, when exclusive breast feeding became the preferred method for the first six months, plus complementary feeding from six months. As alternative feeding method, exclusive formula was recommended if formula was acceptable, feasible, affordable, sustainable and safe (AFASS) [7].

The national PMTCT guidelines were revised in 2007, incorporating the policy changes made by the WHO from 2004 to 2006, while retaining the early recommendations on partner testing [6]. The revised guidelines state that all pregnant women undergoing HIV counselling and testing should be advised to bring their partner for HIV testing [6]. Partner testing and involvement in PMTCT is intended to facilitate the women's coping with their test results, adherence to PMTCT recommendations and to facilitate disclosure [8]. However, the potentials of partner testing in PMTCT settings for the prevention of horizontal transmission of HIV is often neglected. Orne-Gliemann et al. described that the large proportion of new HIV infections is occurring in conjugal relationship [9]. Studies from southern and eastern Africa also reported that the prevalence of HIV sero-discordance is within the range of $36 \%-85 \%[3,10]$. Pregnant women who were HIV negative in early pregnancy could be at risk of acquiring new infection if they had a positive partner at home, which in turn would increase the risk of mother-to-child HIV transmission (MTCT).

In Ethiopia a Monitoring and Evaluation system for HIV/AIDS (M\&E) was first launched in 2003 [11]. The system was established to support and strengthen evidence based performance monitoring for HIV/AIDS related interventions [4]. As part of the M\&E system, monthly PMTCT reports have been collected from different service outlets to evaluate the performance of the national PMTCT programme at facility, sub-city and region level since the launching of the programme. However, despite the availability of comprehensive PMTCT reports the impact of the programme has not been documented. In this study we make use of the available monthly PMTCT reports from the launching of the PMTCT programme in 2004 to August 2009 in order to examine trends in PMTCT service utilization and to assess the rate of MTCT in relation to policy changes.

\section{Methods}

A retrospective study was conducted from May to October 2009. PMTCT monthly reports from February 2004 to August 2009 were reviewed in all the 10 sub-cities of Addis Ababa. The population of Addis Ababa is around three million, and the city is administratively divided into 10 sub-cities and 99 Kebeles. In 2007, a total of 30 hospitals, 29 health centres and 442 clinics were providing health care services [12]. Of the 58 health facilities offering maternity care services, $81 \%$ were providing PMTCT services. In 2007 alone, over 70,000 pregnant women who were eligible for PMTCT services were living in the city. Over $80 \%$ of these pregnant women were attending antenatal care services, yet only $33.1 \%$ of the deliveries were attended by skilled professionals. Of the 24, 584 pregnant women who received HIV counselling and testing in 2007, 7.2\% were HIV positive [12].

The first national PMTCT guidelines were developed in 2001 in preparation to launch a PMTCT programme [4]. In 2004, three years after the development of the first PMTCT guidelines, a free PMTCT programme was launched in selected public health facilities [4]. Initially, only six public health centres were offering PMTCT services across the city. The PMTCT programme continued to expand and from 2007 the programme has been scaled up to private facilities. As of April 2009, of a total of 52 facilities offered PMTCT services, 25 of them being private facilities (unpublished report from Addis Ababa City Administration Health Bureau). All the PMTCT facilities were integrated with Antenatal Care (ANC) service and the majority also provided delivery services.

For the monitoring and evaluation of HIV prevention programmes, a M\&E system was established in 2003. As part of the M\&E system, the monthly PMTCT reporting started in February 2004 with Addis Ketema sub-city. From July 2005, all the sub-cities reported. The PMTCT reporting format developed by the Ministry of Health includes output indicators. The format has been distributed to all service outlets by respective sub-city health 
bureaus. The PMTCT reports were first collected from the log books at the service outlets and then reported to the respective sub-cities by the end of each month. In the sub-cities, the reports from all private and public facilities were compiled and reported to Addis Ababa City Administration Health Bureau. In this study we collected the monthly PMTCT reports from the 10 subcities where they compiled data from all service outlets that started reporting at different times.

The first national PMTCT guidelines was revised in July 2007 to accommodate changes in policies made by the WHO from 2004 to 2006 [6]. Until the revised guidelines came into effect in early 2008, the HIV counselling and testing was offered as an opt-in approach [13], and sdNVP was given to positive women during pregnancy and to their infants within 72 hours of birth [14]. The HIV positive pregnant women were advised to feed exclusive formula if AFASS; if not, exclusive breast feeding [15].

According to the revised guidelines, pregnant women attending ANC should be offered HIV counselling and testing routinely as an opt-out approach $[5,6]$. The HIV positive pregnant women should start ZDV from 28 weeks of gestation plus intrapartum lamivudine and sdNVP followed by ZDV for seven days postpartum. For the infant, ZDV and sdNVP should be given at birth followed by ZDV for seven days if the mother received prophylaxis for at least one month, otherwise the infant should receive ZDV for one month postpartum $[1,6,16]$. The HIV positive pregnant women should receive infant feeding counselling in accordance with the $2006 \mathrm{WHO}$ infant feeding update [6,7]. Moreover, HIV antigen testing of infants with polymerase chain reaction (PCR) at $>=45$ days postpartum replaced the former antibody testing at $>=18$ months of age [6]. Nonetheless, early recommendations on partner testing, irrespective of the woman's HIV status, and referring HIV positive pregnant women for treatment, care and support remained unchanged with renewed emphasis in the revised guidelines [6].

\section{Data analysis}

The reports were entered in Microsoft Excel spreadsheet and analyzed using Pivotal tables and charts. Prevalence and Relative risk (RR) were determined and $\chi^{2}$ test for trend was analysed using the Epicalc 2000 software. The $\chi^{2}$ trend tests were used to check whether there was a linear trend in PMTCT service utilization and HIV prevalence across the years and were reported as RR with associated p-values. The $\chi^{2}$ tests for trend change for all outcome indicators were compared across the years taking 2004 as the reference time. P-value less than 0.05 was considered significant. Proportions are reported with $95 \%$ confidence intervals.
The following outcome indicators were analysed:

1) The proportion of women who received pre-test counselling, HIV testing and post-test counselling among new ANC attendees.

2) The proportion of women who tested positive and partners who received HIV testing among the total number of women who tested for HIV.

3) The proportion of women (and babies) who received ARV prophylaxis, infant feeding counselling and who were referred for treatment, care and support among women who tested positive for HIV.

4) The proportion of babies who tested positive among exposed babies tested for HIV.

\section{Ethical consideration}

The study was reviewed and approved by the Regional Committee for Medical Research Ethics in Western Norway and the Ethical committee of Addis Ababa Region Health Bureau in Ethiopia. Study permit from Addis Ababa Region Health Bureau and respective subcities were obtained.

\section{Results}

We obtained 565 PMTCT monthly reports from the 10 sub-cities. In Lideta sub-city three reports were missing and we collected them from the service outlets instead. In $6.5 \%$ (37) of the reports information on revisiting ANC attendees was either missing, or new and revisit attendees were not reported separately.

During February, 2004 to August, 2009, a total of 663 603 pregnant women attended ANC in health facilities offering PMTCT programme, and 24.6\% (163 635) of them were new attendees. Overall, 135986 women (new and revisit ANC attendees) and 4.9\% (6 664) of their partners received HIV testing and 6.2\% (8 467) of the women were HIV positive. Of the tested women, 97.1\% (131 992) received post-test counselling. Among the HIV positive women, 42.4\% (3 594) received ARV prophylaxis, $41.0 \%$ (3 474) were referred for treatment, care and support and $82.8 \%$ (7 010) received infant feeding counselling. Among babies born to the HIV positive women, 31.0\% (2 621) received ARV prophylaxis and 10.6\% (896) were tested for HIV (See Additional file 1: Compiled data from February, 2004 to August, 2009 from the 10 sub-cities).

\section{Trends in HIV counselling and testing}

Fig 1 presents the year by year trend in HIV counselling and testing utilization. The proportion of women who received pre-test counselling, testing and post-test counselling among new ANC attendees was 66.3\% (95\% CI 64.8-67.7) in 2004 based on the reports from the three sub-cities that started reporting in 2004. In 2005, following the PMTCT programme scale up to the rest of the 


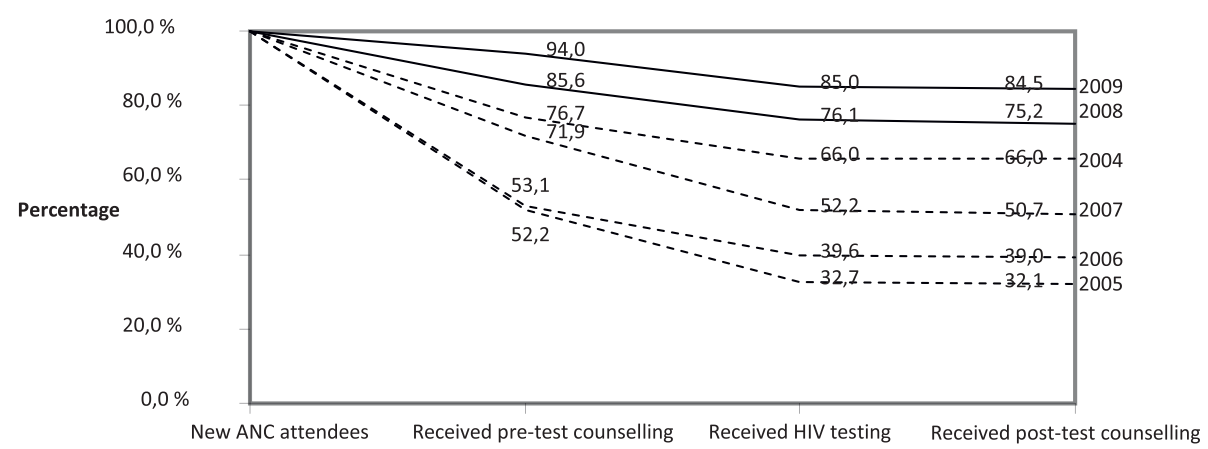

Figure 1 Proportion of women who received pre-test counselling, testing and post-test counselling among new ANC attendees. - In 2004 to 2007 when the HIV testing was offered in an opt-in approach. In 2008 and 2009 when the HIV testing was offered routinely in an opt-out approach.

sub-cities, the counselling and testing utilization dropped to $32.1 \%$ (95\% CI 31.5-32.7). Although the drop appeared to be marked in the three sub-cities that started reporting earlier, those that started later also showed a similar trend. The poor utilization persisted till 2007 when only 50.7\% (95\% CI 50.2-51.2) of the new attendees received HIV counselling and testing. The PMTCT programme gained momentum in 2008 when the revised guidelines that incorporated routine opt-out testing offer came into effect. Utilization of HIV counselling and testing increased to $84.5 \%$ (CI 84.1-84.9) in 2009. The trend in receiving posttest counselling remained stable at high level across the years which imply that almost all the tested women collected their HIV test result (Fig 1).

There were variations across the sub-cities in the proportion of women who had not received pre-test counselling or testing. In 2009, one year following the shift to routine opt-out approach, $12.0 \%, 18.4 \%, 19.3 \%, 14.0 \%$, $14.6 \%$ and $27.3 \%$ of the women attending ANC in Addis Ketema, Arada, Bole, Kirkos, Lideta and Nifas Silk Lafto sub-city, respectively, did not receive pre-test counselling. Similarly, among women who received pre-test counselling in 2009, 17.3\%, 11.3\%, 17.0\% and 24.8\% in Arada, Bole, Gulele and Kirkos sub-cities, respectively, did not receive HIV testing.

\section{HIV testing versus HIV prevalence}

Fig 2 presents the overall trends in HIV prevalence among ANC attendees across the years. The HIV prevalence among the total ANC attendees (both new and revisit) who tested for HIV appeared to decline steadily from $10.5 \%$ (CI $9.6-11.5$ ) in 2004 to $4.6 \%$ (95\% CI 4.3 4.8) in 2009 (RR 0.46) in parallel with the increasing number of women testing for HIV. During the same period the trends in HIV prevalence across the subcities showed significant decline, except Yeka sub-city where the HIV prevalence actually increased by $17 \%$ in 2009 compared to 2004 (RR 1.17, p < 0.05) (Table 1).

\section{ARV Prophylaxis uptake}

Of the 8467 HIV positive women, ARV prophylaxis was given to $42.4 \%$ (95\% CI 41.4-43.5) and to $31.0 \%$ (95\% CI $30.0-31.0$ ) of their infants. From early 2008, the prophylaxis regimen was shifted from sdNVP to multidrug ZDV regimen. Despite the big difference in the two regimens, the reports did not show marked changes of the trend in prophylactic ARV uptake. In 2004, the ARV prophylaxis uptake by the women and infants was low, but the gap in prophylaxis uptake between the women $(24.8 \% .95 \% \mathrm{CI}$ 20.8-29.2) and babies (23.2\%. 95\% CI 19.3-27.5) was narrow. In 2005 and 2006 an increased proportion of women received prophylaxis, $41.6 \%$ (95\% CI 39.0-44.3) and $55.0 \%$ (95\% CI 52.3-57.4) respectively. In 2006 the proportion and number of women who received ARV prophylaxis peaked, yet only $35.1 \%$ (95\% CI $32.8-37.4$ ) of their infants received prophylaxis, resulting in the widest gap in prophylaxis uptake. In 2007, the prophylaxis uptake by the women and infants dropped to $33.8 \%$ (95\% CI 31.6-36.1) and 25.4\% (95\% CI 23.4-27.5), respectively, and the gap narrowed again. The prophylaxis uptake shows an increasing trend since 2008 (Fig 3).

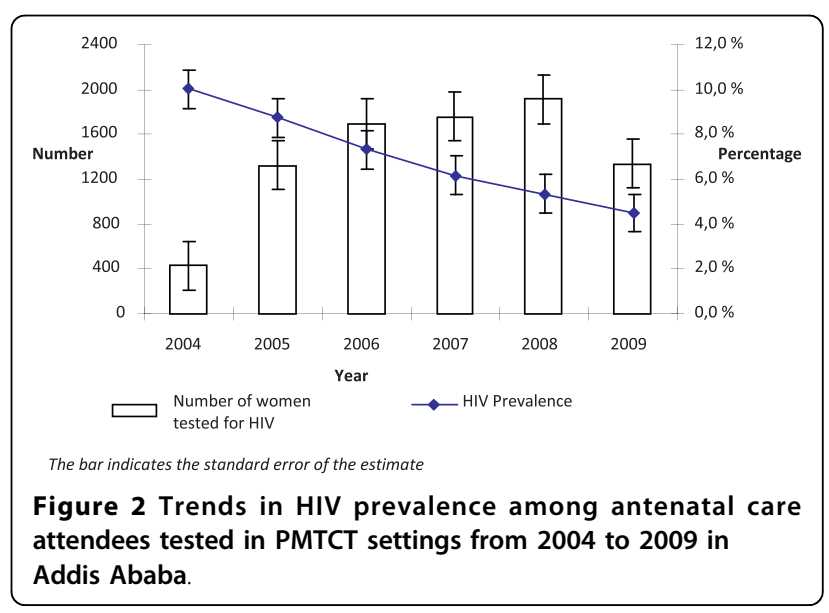


Table 1 HIV prevalence among antenatal care attendees who tested for HIV, relative risk and $\chi^{2}$ for trend test across the years by sub-city

\begin{tabular}{|c|c|c|c|c|c|c|c|c|c|c|c|c|c|}
\hline \multirow[t]{2}{*}{ Sub-city } & \multicolumn{2}{|c|}{2004} & \multicolumn{2}{|c|}{2005} & \multicolumn{2}{|c|}{2006} & \multicolumn{2}{|c|}{2007} & \multicolumn{2}{|c|}{2008} & \multicolumn{2}{|c|}{2009} & \multirow[t]{2}{*}{$\chi^{2}$ trend } \\
\hline & $\%(n)$ & RR & $\%(n)$ & RR & $\%(n)$ & RR & $\%(n)$ & $\mathrm{RR}$ & $\%(n)$ & RR & $\%(n)$ & $\mathrm{RR}$ & \\
\hline Addis Ketema & $12.8(195)$ & Ref & 11.1(168) & 0.99 & 7.4(170) & 0.66 & $7.8(231)$ & 0.70 & $4.6(178)$ & 0.41 & $4.7(130)$ & 0.42 & $127.3 *$ \\
\hline Akaki Kaliti & & & $8.7(99)$ & Ref & $7.0(131)$ & 0.81 & $6.8(16)$ & 0.78 & $4.9(136)$ & 0.57 & $5.3(113)$ & 0.61 & $22.3 *$ \\
\hline Arada & & & $8.9(134)$ & Ref & $8.2(185)$ & 0.92 & $6.3(159)$ & 0.71 & $5.9(298)$ & 0.66 & $3.8(123)$ & 0.43 & $64.4^{*}$ \\
\hline Bole & 8.6(89) & Ref & $10.0(112)$ & 1.17 & $6.8(149)$ & 0.79 & $5.5(158)$ & 0.64 & $4.8(171)$ & 0.56 & $3.2(130)$ & 0.37 & $105.4^{*}$ \\
\hline Gulele & & & $6.7(77)$ & Ref & $6.6(118)$ & 0.98 & $5.7(156)$ & 0.84 & $5.0(136)$ & 0.75 & $4.5(93)$ & 0.67 & $12.1^{* *}$ \\
\hline Kirkos & & & $10.2(89)$ & Ref & $9.5(188)$ & 0.93 & $7.1(162)$ & 0.69 & $6.8(191)$ & 0.66 & $4.6(122)$ & 0.44 & $54.3 *$ \\
\hline Kolfe Keraniyo & & & $5.4(67)$ & Ref & $4.9(75)$ & 0.91 & $2.8(70)$ & 0.51 & $3.3(73)$ & 0.62 & $3.7(81)$ & 0.69 & $7.7^{\ddagger}$ \\
\hline Lideta & $9.6(148)$ & Ref & $10.2(244)$ & 1.06 & 6.8(198) & 0.71 & $5.9(228)$ & 0.62 & $4.8(246)$ & 0.50 & $4.6(217)$ & 0.48 & $119.8^{*}$ \\
\hline Nifas-silk Lafto & & & $8.0(175)$ & Ref & $7.5(253)$ & 0.93 & $6.0(232)$ & 0.74 & $4.6(217)$ & 0.57 & $4.0(140)$ & 0.49 & $70.5^{*}$ \\
\hline Yeka & & & $7.9(161)$ & Ref & 7.6(228) & 0.96 & $8.2(206)$ & 1.03 & $9.3(268)$ & 1.17 & $9.2(193)$ & 1.17 & $6.2^{\ddagger}$ \\
\hline
\end{tabular}

Ref $=$ Reference ${ }^{*} P<0.0001{ }^{* *} P<0,001 \neq P<0,01 \neq \neq P<0,05$ NB: The denominator for each cell is different

\section{Referral for treatment, care and support}

The need for referring HIV positive pregnant women for treatment, care and support gained momentum in response to the 2004-2006 national roadmap to accelerate access to antiretroviral therapy [17] and the launching of the revised PMTCT guidelines [6]. The proportion of HIV positive pregnant women referred for treatment, care and support increased from 3.2\% (95\% CI 1.9-5.5) in 2004 to $59.9 \%$ (95\% CI 57.2-62.5) in 2009. In other words, the HIV positive pregnant women in 2009 were over 18 times more likely to be referred for treatment, care and support than their counterparts in 2004 (RR $18.5 \mathrm{X}^{2}$ trend $\left.\mathrm{p}<0.0001\right)$.

\section{Infant feeding counselling}

In 2004, when the PMTCT programme was first launched, 58.3\% (95\% CI 53.5-63.0) of the HIV positive women received infant feeding counselling, compared to 87.3\% (95\% CI 85.3-89.0) in 2009. The HIV positive women in 2009 had a $50 \%$ more chance to receive infant feeding counselling than the positive women in 2004 (RR 1.5, $\chi^{2}$ trend $\left.p<0.001\right)$. Despite the changes in infant feeding recommendation at different times, the report lack information on the kind of infant feeding advice given, infant feeding choice made by the women or pattern of infant feeding.

\section{HIV infection among exposed babies}

Overall, only $10.6 \%$ (896) (95\% CI 9.9-11.2) of the HIV positive women completed their follow up to child HIV testing. The rate of MTCT was evaluated based on the 896 babies tested for HIV during the period 2006-2009. Among the 896 exposed babies 106 were HIV positive. Of the babies on sdNVP regimen who tested at $>=18$ months with rapid antibody test, $14.3 \%$ (95\% CI 7.9224.0 ) in 2006 and $15.0 \%$ (95\% CI 9.8-22.1) in 2007 were HIV positive. In 2009, among babies on the multidrug ZDV regimen who tested at $>=45$ days postpartum with

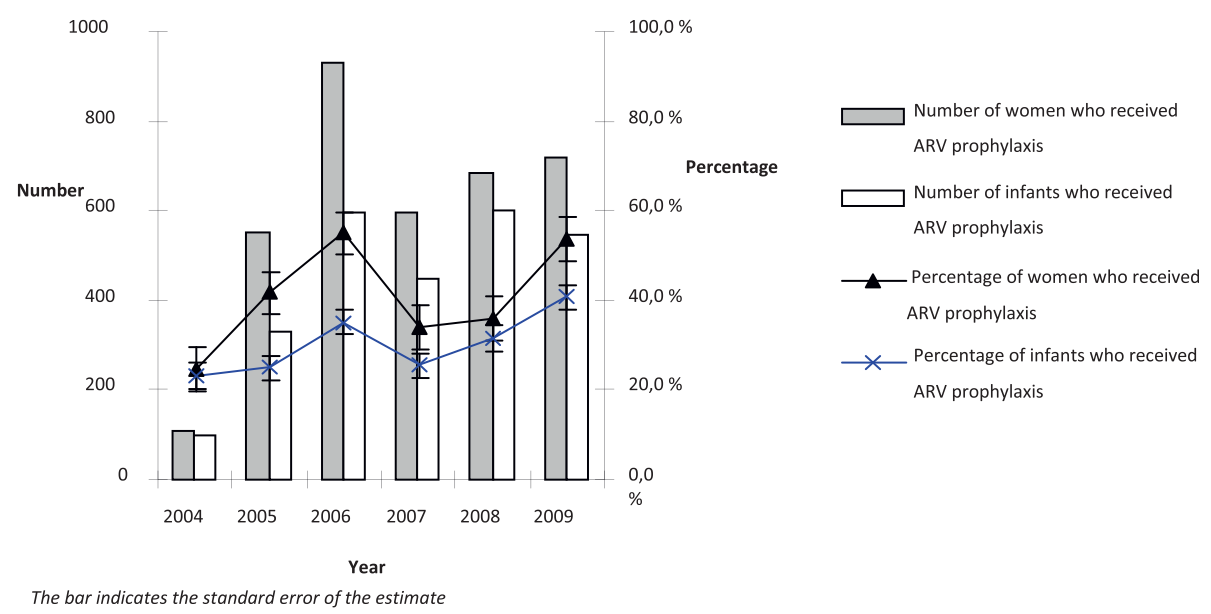

Figure 3 Percentage of women and babies who received ARV prophylaxis from 2004 to 2009 in Addis Ababa. 


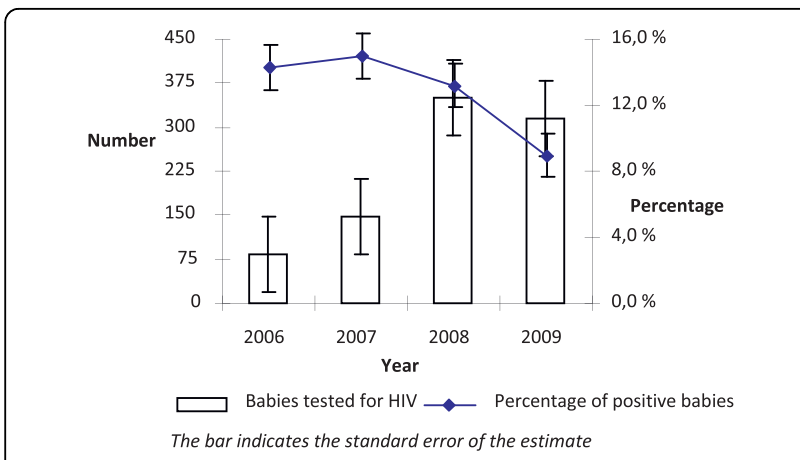

Figure 4 HIV infection among exposed babies tested for HIV from 2006 to 2009 in Addis Ababa.

antigen test using PCR, 8.2\% (95\% CI 5.55-11.97) were HIV positive (Fig 4).

\section{Partner testing}

The overall trend in the proportion of partners tested among women who received HIV testing remained stable at low level i.e 6.4\% (95\% CI 5.7-7.2) in 2004 and 5.3\% (95\% CI 5.0-5.5) in 2009. Compared to the women who were tested in 2004, women tested in 2009 were $14 \%$ less likely to be tested with their partner (RR $0.86, \mathrm{p}<0.01)$. However, there was an increase in the absolute number of partners tested across the years in parallel with the increased number of women who tested positive. In 2009 the number of partners tested was higher than the number of women who tested positive (Fig 5).

\section{Discussion}

In this study we examined trends in PMTCT service utilization and assessed the rate of MTCT in Addis Ababa,
Ethiopia. The HIV counselling and testing service utilization improved substantially in 2009 following policy shift to routine opt-out approach. The HIV prevalence appeared to decrease steadily paralleling the increased number of women tested for HIV. Irrespective of policy changes, the uptake of ARV prophylaxis and loss to follow up remained unimproved. Out of the 10.6\% (896) exposed babies tested for HIV, the cumulative probability of HIV infection decreased to $15.0 \%$ by 2007 among babies on sdNVP regimen tested at $>=18$ months of age, and to $8.2 \%$ by 2009 among infants on ZDV regimen tested at $>=45$ days of age. The proportion of HIV positive pregnant women referred for treatment, care and support increased eighteen-fold in 2009 compared to 2004. Meanwhile, the proportion of partners tested in the PMTCT setting declined significantly by $14 \%$ from 2004 to 2009.

The proportion of women who received HIV counselling and testing among new ANC attendees increased significantly from $50.7 \%$ in 2007 to $84.5 \%$ in 2009 following the shift to routine opt-out testing. Consistent with our findings, studies from other resource poor settings have revealed significant improvement in HIV testing at ANC from $45 \%$ to $99 \%$ following the shift to routine opt-out approach [18-20]. Nevertheless, over $10 \%$ of the new ANC attendees had not received pretest counselling or testing in 2009. In this regard the opt-out approach, which seems to be robust to maximise HIV testing in many settings [18-20], did not show remarkable success in our study. This could be attributed to two major reasons. First, most of the studies that reported nearly a $100 \%$ HIV test acceptance when testing was offered routinely as an opt-out approach, were pilot initiatives [18-20], unlike our study that compiled

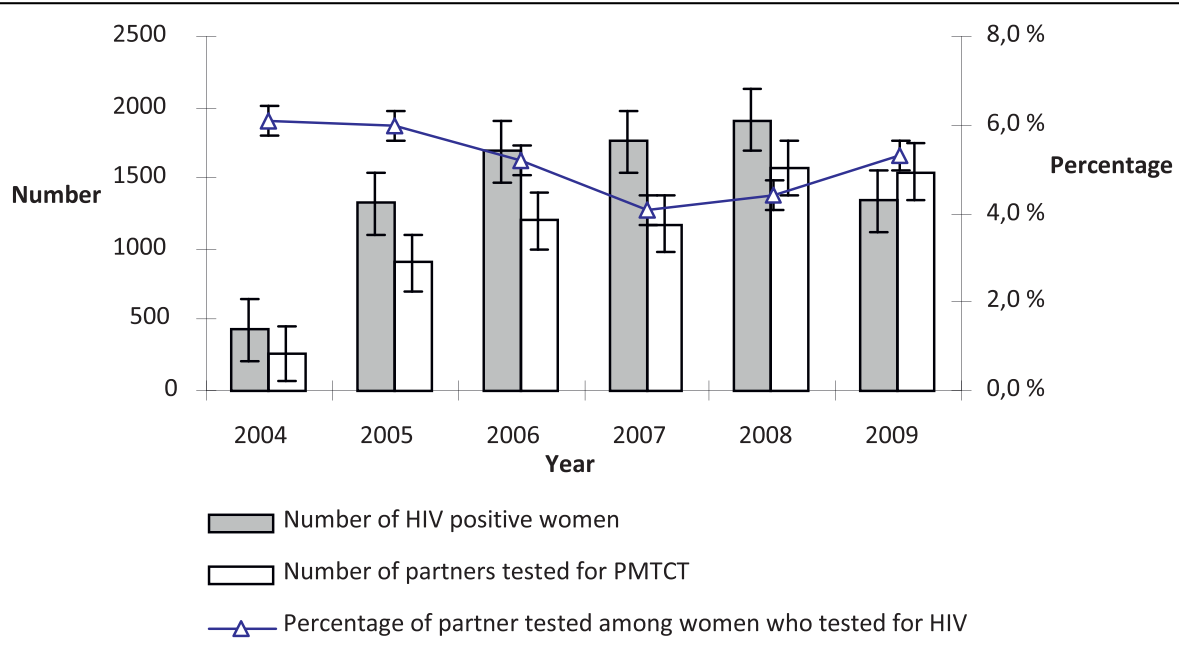

The bar indicates the standard error of the estimate

Figure 5 Trends in partner testing in the PMTCT settings from 2004 to 2009 in Addis Ababa. 
PMTCT reports from a national programme. In line with our argument, a study in Kenya based on data from 43 PMTCT service outlets reported a $80.6 \% \mathrm{HIV}$ test acceptance [21]. Second, the expansion of the PMTCT programme to private facilities seem to have contributed to the persistence of a large proportion of women who had not received HIV counselling or testing even after the shift to routine testing. Site factors are reported to be more relevant than participant factors in determining HIV test acceptance in a study from Kenya [21]. In particular, characteristics of the provider appear to be an important determinant for HIV test acceptance. For instance, characteristics of the midwives were found to be an independent determinant for HIV testing uptake in England [22], whereas high test refusal was associated with HIV testing being offered by general practitioners in Canada [21,23]. In almost all the private facilities in Addis Ababa, ANC and HIV testing was offered by physicians who had little or no training on HIV counselling and testing in an opt-in approach. This indicates that there are gaps in the implementation of the routine opt-out testing strategy, particularly in private facilities. On top of that, the utilization of post-test counselling and collection of test result by almost all tested women throughout the years, irrespective of the testing approach employed, implies that the persistent gap in pre-test counselling and HIV testing service utilization could be an evidence of failure of the health system to deliver the programme rather than a failure of the women.

The HIV prevalence among the tested women had reduced by $54 \%$ in 2009 compared to the level in 2004, in parallel to the increased number of women being tested. Similar declining trend in HIV prevalence has been observed among adults and from a sentinel surveillance report in Ethiopia. Synergy between the natural progression of the HIV epidemic and behaviour changes among the general population in terms of increased condom use and reduced risky sexual behaviour seem to contribute to the declining prevalence $[3,4]$. The HIV prevalence estimate in our study was lower than that of the ANC sentinel surveillance report, i.e $6.2 \%$ vs $9.3 \%$ in $2007 / 2008$ [12]. Consistent with our finding, a study that compiled data from Kenya, Ethiopia, and Zimbabwe for 2005 reported that the HIV prevalence in Ethiopia was $6.4 \%$ from PMTCT programme reports and $8.2 \%$ from sentinel surveillance reports [24-26]. The two reports, although generated from the same population, have differences in population size and timing of data collection [24-26]. The PMTCT report includes all women who participated in the PMTCT programme all year round. In the sentinel surveillance, leftover blood samples were collected from a smaller number of ANC attendees from selected facilities, often for a period of 3 months biennially.
The decreasing trend in the HIV prevalence estimate across the years could also be attributed to a more representative sample of pregnant women tested for HIV in our study. In other words, the inclusion of more service outlets and therefore reaching out to a large population in our study seems to be more representative, unlike the sentinel surveillance report that relied on reports from a few public facilities. Even in the sentinel surveillance reports, as the number of sentinel sites increased the estimated HIV prevalence declined [4]. More importantly we included reports from private facilities where the HIV prevalence is reported to be lower compared to ANC attendees in public facilities, 2.4\% (unpublished report from Addis Ababa City Administration Health Bureau) vs 9.3\% [12] respectively in $2007 / 2008$. Moreover, the HIV prevention potential of Highly Active Antiretroviral Therapy (HAART) should not be ignored [27].

Despite the marked increase in HIV testing following the shift to routine opt-out approach, neither the proportion nor the number of women receiving ARV prophylaxis have increased. In 2009 , only $53.7 \%$ of the women and $40.7 \%$ of their babies received ARV prophylaxis. Consistent with our findings, in Addis Ababa, $49.3 \%$ of the HIV positive women and $35.3 \%$ of their babies received ARV prophylaxis in 2007/2008 [28] and the programme achieved only $30 \%$ compared to $80 \%$ as the target [12]. Actually, poor ARV prophylaxis uptake is not a new story, even those intervention studies that show almost $100 \%$ successes in HIV testing were in short of ARV prophylaxis uptake [18-20]. However, the lack of improvement in ARV prophylaxis utilization following the shift to routine testing is an issue of great concern as the shift to an opt-out approach was primarily intended to increase the proportion of women and infants receiving prophylactic ARV drugs [5]. In the era of routine testing for HIV, the PMTCT programme appears to be an effective screening programme than a prevention programme due to the large number of dropouts after testing. Yet, the gain in HIV testing turnout limits the weakness of the routine opt-out approach in terms of subsequent dropouts and poor adherence to ARV prophylaxis [29]. Currently the availability of potent ARV prophylaxis is changing the landscape, and even mother-to-child HIV transmission through breast feeding has become less of a concern [30]. Yet, still more lives are at stake because of the lack of improvement in uptake of this critical component of the PMTCT programme.

According to Kasenga et al., skilled attendance at birth is an important determinant of ARV prophylaxis uptake that requires thorough consideration [31]. In Addis Ababa, only $30 \%$ of the pregnant women had skilled attendance at birth in 2008 [12] corresponding to the 
proportion of infants who received ARV prophylaxis [28]. Since infants are given prophylaxis within 72 hours of birth at the health facility, infants delivered at home have less chance to receive ARV prophylaxis than infants delivered in health facilities. An intervention study from Zambia gives some hope that dropouts and non-adherence to ARV prophylaxis could be reduced to zero. This study, which employed multiple interventions, increased the ARV prophylaxis uptake from $29 \%$ at baseline to $100 \%$ within 3 years [32]. This gives reassurance that the official $80 \%$ ARV prophylaxis uptake goal for Ethiopia could be achieved through concerted effort and renewed commitment.

According to the findings, an increasing proportion of HIV-positive pregnant women were referred for treatment, care and support services, from $3.2 \%$ in 2004 to $59.9 \%$ in 2009 . The referral is intended for prompt initiation of ARV prophylaxis or treatment for eligible women using $\mathrm{CD}_{4}$ or lymphocyte count and WHO staging criteria [6]. By doing so, the programme is addressing the most important issues that PMTCT programmes have been criticized for, i.e the lack of sensitivity to the needs of pregnant women. The PMTCT programme is increasingly successful in bridging the gap between prevention and treatment to address the moral and ethical questions raised over the years [1]. The eighteen-fold increase in the proportion of positive pregnant women being referred for treatment, care and support from 2004 to 2009 demonstrates the good will and the potential to integrate new policies and strategies. Yet, more has to be done to ensure that all HIV-positive pregnant women have access to prophylaxis or treatment, care and support.

The ultimate objective of a PMTCT programme is to avert new HIV infections among children. However, only $10.6 \%$ (896) of the HIV positive pregnant women completed their follow up to infant HIV testing. The rates of MTCT were therefore assessed based on test result of the 896 babies tested for HIV. In the absence of any PMTCT intervention the cumulative probability of HIV infection among exposed babies aged $>=18$ months is in the range of $29 \%$ to $47 \%$ according to a cohort study conducted in an orphanage in Addis Ababa [4]. In our study, the cumulative probability of HIV infection among exposed babies on sdNVP regimen tested at $>=18$ months was $14.3 \%$ in 2006 and $14.9 \%$ in 2007. According to the HIVNET 012 randomized trial, sdNVP regimen has a $41 \%$ efficacy. In this trial, consistent with our findings, the cumulative probability of infant HIV infection is $15.7 \%$ among breast fed infants tested at $>=18$ months [14]. However, a methodologically similar study from Malawi that compiled monthly reports showed a $15.5 \%$ HIV infection rate among infants on sdNVP regimen tested at 6 weeks postpartum without accounting for the breast feeding transmission [33]. Nevertheless, there is a possibility that our estimate could be biased due to the large loss to follow up.

In 2009, 8.2\% of the exposed infants on ZDV regimen tested at 45 days were HIV positive. In the Petra clinical trial, multidrug ZDV regimen showed 63\% efficacy in reducing MTCT. In this trial the rate of HIV transmission among infants on multidrug ZDV regimen tested at 45 days was $5.7 \%$ [16]. Considering the fact that our data are generated from a national PMTCT programme and the obvious methodological difference with the Petra trial, the $8.2 \%$ infant infection rate reported in our study indicates the success of the national PMTCT programme among those who completed their follow up to infant HIV testing. A cohort study from similar resource poor settings that evaluated the effectiveness of a PMTCT programme among predominantly formula fed infants on ZDV regimen tested at $>=45$ days reported a 9.1\% cumulative infant HIV infection, higher than the rate of HIV infection reported in our study [34]. However, since the HIV testing was done at $>=45$ days, those HIV negative infants who continue to breast feed are still at risk of acquiring new infection. In general, the rate of MTCT averted by the national PMTCT programme appears promising among those who adhered to the programme. Nevertheless, the possibility of underestimation cannot be excluded since we lack information on loss to follow up. In line with this limitation, Ahoua et al. found that the cumulative probability of infant HIV infection among tested infants was $8.3 \%$, whereas it was $15.5 \%$ when HIV related deaths were included in the analysis [35].

The last, but not least important PMTCT programme outcome indicator examined in our study was partner testing. The proportion of partners tested remained very low with a $14 \%$ significant decline in 2009 compared to 2004. A review on couple centred counselling shows that partner involvement in HIV testing not only helps to increase disclosure, condom use and uptake of ARV prophylaxis but also contributes to the lower rate of seroconversion compared to individual counselling [8]. We noted a parallel trend in the number of partners tested with the number of women who tested positive across the years. This indicates that most of the partners who came for HIV testing were those whose wives tested positive. Partner testing in the context of PMTCT seems to facilitate women's coping, yet missing out the important prevention aspect by not advising HIV negative women to bring their partner for testing.

A study from South Africa shows that 3\% of the pregnant women who were found to be HIV negative in their first HIV testing during pregnancy became HIV positive in repeat test in late pregnancy, giving a $10.7 \%$ incidence per year [36]. This indicates that women are 
at risk to acquire new HIV infection from their HIV positive partner anytime during pregnancy and even during breast feeding. In eastern and southern African region, including Ethiopia, 36-85\% of HIV positive individuals are believed to live with an HIV negative partner $[4,10]$. Discordant couples are the newly identified high risk group in Ethiopia, as most infections are occurring within marriage. Because of mucosal and hormonal changes during pregnancy, the HIV incidence is four times higher among pregnant women compared to their non-pregnant counterparts [37]. Meanwhile, women having recent HIV infection are more likely to transmit HIV infection to their babies [36]. Therefore, it is crucial to focus on partner testing and involvement in the PMTCT programme to optimise programme effectiveness. The current strategies in Addis Ababa, that include giving priority for women coming with their partner for testing and sending an invitation home to partner should be encouraged.

One of the limitations of our study is that the rate of MTCT was examined based on infants tested for HIV, which could actually be underestimated due to the large loss to follow up. Considering the lack of a system to trace loss to follow up, our finding still highlights the potentially averted infections. Another limitation is that the PMTCT programme reached out to only $80 \%$ of pregnant women due to incomplete ANC attendance [12] and the findings seem not to represent the whole nation, where a high proportion of the population is rural. However, since it was generated from a national programme, lessons learnt herein could benefit the PMTCT programme across the country. We also believe that our findings are generalisable to the big cities where the HIV prevalence is higher.

Retrospective data collected primarily for reporting purposes always have weakness, especially in resource poor settings where the quality of reports are often questionable [11]. By limiting our study objectives to those indicators that could be calculated from the reports we minimized the risk of having incomplete data. We obtained almost all the reports from February 2004 to August 2009 from the 10 sub-cities. To validate our data we checked Addis Ababa City Administration Health Bureau and NGO reports, and our data were found to be consistent. For missing reports we obtained data from log books and reports at service outlets.

\section{Conclusion}

Our findings suggest that the proportion of women receiving HIV counselling and testing has increased substantially from 2004 to 2009. The shift in HIV testing approach has further reduced the proportion of women not receiving HIV counselling and testing. However, the lack of improvement in ARV prophylaxis uptake after the shift to routine opt-out testing is an issue of great concern as the shift was intended to facilitate access to ARV prophylaxis. The health system appeared not only to fail to deliver the most critical component of the PMTCT programme, i.e ARV prophylaxis, but also to retain the HIV positive women to complete their follow up. The effectiveness of the national PMTCT programme is questionable as only $10 \%$ of the HIV positive women completed their follow up to infant HIV testing. A tracing system for loss to follow up should be in place for measuring the actual impact of the programme. In addition, the sub-cities and the Addis Ababa city Administration Health Bureau must devise a mechanism to increase prophylaxis uptake and to retain the HIV positive women in the programme until the follow up is completed. The counselling Training should emphasize partner testing to ensure that all women tested in PMTCT settings receive counselling on partner testing, irrespective of their HIV status. Further research is imperative to identify and address challenges to ARV prophylaxis uptake, partner testing, referral for treatment, care and support and challenges to follow up. We recommend the health facilities, sub-cities and the City Administration Health Bureaus to utilize the monthly reports to identify gaps in service utilization and to come up with context specific solutions for the realisation of the country's goal of "HIV free generation by $2020 "$.

\section{Additional material}

Additional file 1: Compiled monthly PMTCT reports from February, 2004 to August, $\mathbf{2 0 0 9}$ for the $\mathbf{1 0}$ Sub-cities in Addis Ababa. The table containes monthly PMTCT reports from February, 2004 to August 2009 collected from the 10 sub-cities. Addis Ketema, Bole and Lideta sub-cities started reporting from 2004 while the rest of the sub-cities reported from 2005 .

\section{Acknowledgements}

This study was financially supported by the Centre for International Health, University of Bergen, the Meltzer Foundation and Statens Lånekasse, Norway. We thank the Addis Ababa City Administration Health Bureau and the health bureaus' in the respective sub-cities for permitting us to collect the reports. Both Addis Ababa City Administration Health Bureau and Intra Health deserve acknowledgement for providing their PMTCT reports for validation. Moreover, we are grateful to Karen Marie Moland for revising the manuscript and to Addis Mulu for transferring the data into a pivotal table and chart format.

Author details

${ }^{1}$ Centre for International Health, University of Bergen, Overlege Danielssens Hus, Årstav. 21, Bergen 5020, Norway. ${ }^{2}$ College of Medical and Health Sciences, Department of Nursing and Midwifry, Hawassa University, Awassa, p.o.box 1560, Ethiopia.

\section{Authors' contributions}

AHM prepared the study proposal, collected and analyzed the data, interpreted the findings and wrote the manuscript. OM was involved in developing the study proposal, supervising the data collection and revising the manuscript. SGH was involved in developing the study proposal and 
revising the manuscript. All authors have read and approved the final manuscript.

\section{Competing interests}

The authors declare that they have no competing interests.

Received: 9 April 2010 Accepted: 9 September 2010

Published: 9 September 2010

\section{References}

1. WHO: Antiretroviral drugs for treating pregnant women and preventing HIV infection in infants: towards universal access: recommendations for a public health approach.- 2006 version. WHO: Geneva 2006.

2. Letvin NL: Virology. Moving forward in HIV vaccine development. Science (New York, N.Y.) 2009, 326(5957):1196-8.

3. Potts $M$, et al: Public health. Reassessing HIV prevention. Science (New York, N.Y.) 2008, 320(5877):749-50.

4. HAPCO/GAMET: HIV/AIDS in Ethiopia - An Epidemiological Synthesis. Washington DC 2008

5. UNAIDS/WHO: UNAIDS/WHO Policy statement on HIV testing WHO: Geneva 2004.

6. HAPCO/FMOH: Guidelines For Prevention of Mother-to-Child Transmission of HIV in Ethiopia MOH: Addis Ababa 2007

7. WHO, et al: HIV and infant feeding: update based on the technical consultation held on behalf of the Inter-agency Task Team (IATT) on Prevention of HIV infections in pregnant women, Mothers and their Infants, Geneva, 25-27 October 2006 WHO: Geneva 2007, iv, 14 p.

8. Desgrées-du-Loû A, Orne-Gliemann J: Couple-centred testing and counselling for HIV serodiscordant heterosexual couples in sub-Saharan Africa. Reproductive health matters 2008, 16(32):151-61.

9. Orne-Gliemann J, et al: Couple-oriented prenatal HIV counseling for HIV primary prevention: an acceptability study. BMC public health 2010, 10:197.

10. Lingappa $J R$, et al: Regional differences in prevalence of HIV-1 discordance in Africa and enrollment of HIV-1 discordant couples into an HIV-1 prevention trial. PloS one 2008, 3(1):e1411.

11. FMOH: Health Managment Information System (HMIS)/Monitoring and Evaluation (M\&E); Strategic Plan for Ethiopian Health Sector. FMOH: Addis Ababa 2008.

12. FMOH: Health and health related indicators FMOH: Addis Ababa 2008.

13. CDC: U.S. Public Health Service Recommendations for Human Immunodeficiency Virus Counseling and Voluntary Testing for Pregnant Women MMWR 1995, 44(RR-7).

14. Jackson JB, et al: Intrapartum and neonatal single-dose nevirapine compared with zidovudine for prevention of mother-to-child transmission of HIV-1 in Kampala, Uganda: 18-month follow-up of the HIVNET 012 randomised trial. Lancet 2003, 362(9387):859-68.

15. WHO, et al: HIV and infant feeding: guidelines for decision-makers. WHO: Geneva 2003, 75.

16. Petra Study Team: Efficacy of three short-course regimens of zidovudine and lamivudine in preventing early and late transmission of HIV-1 from mother to child in Tanzania, South Africa, and Uganda (Petra study): a randomised, double-blind, placebo-controlled trial. Lancet 2002, 359(9313):1178-86

17. FMOH: Accelarating access to HIV/AIDS treatment in Ethiopia: Road Map for 2004-2006. FMOH: Addis Ababa 2005.

18. Kasenga $F$, et al: The implications of policy changes on the uptake of a PMTCT programme in rural Malawi: first three years of experience. Global health action 2009, 2.

19. Chandisarewa W, et al: Routine offer of antenatal HIV testing ("opt-out" approach) to prevent mother-to-child transmission of HIV in urban Zimbabwe. Bulletin of the World Health Organization 2007, 85(11):843-50.

20. Creek $T L$, et al: Successful introduction of routine opt-out HIV testing in antenatal care in Botswana. Journal of acquired immune deficiency syndromes (1999) 2007, 45(1):102-7.

21. Anand $A$, et al: Site factors may be more important than participant factors in explaining HIV test acceptance in the prevention of motherto-child HIV transmission programme in Kenya, 2005. Tropical medicine \& international health: TM \& IH 2009, 14(10):1215-9.

22. Simpson WM, et al: $\mathrm{A}$ randomised controlled trial of different approaches to universal antenatal HIV testing: uptake and acceptability and Annex:
Antenatal HIV testing - assessment of a routine voluntary approach. Health technology assessment (Winchester, England) 1999, 3(4):1-112.

23. Wang FL, et al: Potential factors that may affect acceptance of routine prenatal HIV testing. Canadian journal of public health Revue canadienne de santé publique 2005, 96(1):60-4

24. Fabiani $M$, et al: Using prevalence data from the programme for the prevention of mother-to-child-transmission for HIV-1 surveillance in North Uganda. AIDS (London, England) 2005, 19(8):823-7.

25. Mpairwe $\mathrm{H}$, et al: HIV risk perception and prevalence in a program for prevention of mother-to-child HIV transmission: comparison of women who accept voluntary counseling and testing and those tested anonymously. Journal of acquired immune deficiency syndromes (1999) 2005, 39(3):354-8.

26. Bolu $\mathrm{O}$, et al: Utility of antenatal HIV surveillance data to evaluate prevention of mother-to-child HIV transmission programs in resourcelimited settings. American journal of obstetrics and gynecology 2007, 197(3): S17-25.

27. Cohen J: 17th Conference on Retroviruses and Opportunistic Infections, 16-19 February, San Francisco, CA. Treatment as prevention. Science (New York, N.Y.) 2010, 327(5970):1196-7.

28. HAPCO: Annual HIV/AIDS Monitoring and Evaluation Report, HAPCO, Editor $\mathrm{MOH}$ : Addis Ababa 2008

29. Manzi $M$, et al: High acceptability of voluntary counselling and HIVtesting but unacceptable loss to follow up in a prevention of mother-tochild HIV transmission programme in rural Malawi: scaling-up requires a different way of acting. Tropical medicine \& international health: TM \& IH 2005, 10(12):1242-50.

30. WHO: New WHO recommendations: Preventing mother-to-child transmission WHO: Geneva 2009.

31. Kasenga F, Hurtig AK, Emmelin M: Home deliveries: implications for adherence to nevirapine in a PMTCT programme in rural Malawi. AIDS care 2007, 19(5):646-52.

32. Kwasi Torpey MK, Kasonde Prisca, Dirks Rebecca, Bweupe Maxmillian, Thompson Catherine, Mukadi Diul: Increasing uptake of prevention of mother-to-child transmission of HIV services in a resource-limited setting. BMC Health Services Research 2010, 10(29).

33. Moses A, et al: Prevention of mother-to-child transmission: program changes and the effect on uptake of the HIVNET 012 regimen in Malawi. AIDS (London, England) 2008, 22(1):83-7.

34. Noel F, et al: Improving outcomes in infants of HIV-infected women in a developing country setting. PloS one 2008, 3(11):e3723.

35. Ahoua $L$, et al: Evaluation of a 5-year programme to prevent mother-tochild transmission of HIV infection in Northern Uganda. Journal of tropical pediatrics 2010, 56(1):43-52.

36. Moses SE, et al: HIV-1 seroconversion during pregnancy resulting in vertical transmission. Journal of clinical virology: the official publication of the Pan American Society for Clinical Virology 2008, 41(2):152-3.

37. Moodley $D$, et al: High HIV incidence during pregnancy: compelling reason for repeat HIV testing. AIDS (London, England) 2009, 23(10):1255-9.

\section{Pre-publication history}

The pre-publication history for this paper can be accessed here: http://www.biomedcentral.com/1472-6963/10/267/prepub

\section{doi:10.1186/1472-6963-10-267}

Cite this article as: Mirkuzie et al:: Promising outcomes of a national programme for the prevention of mother-to-child HIV transmission in Addis Ababa: a retrospective study. BMC Health Services Research 2010 10:267. 